\title{
miRNA-22I of exosomes originating from bone marrow mesenchymal stem cells promotes oncogenic activity in gastric cancer
}

This article was published in the following Dove Press journal:

OncoTargets and Therapy

22 August 2017

Number of times this article has been viewed

\author{
Min Ma, ${ }^{1, *}$ Shilin Chen, ${ }^{1, *}$ \\ Zhuo Liu,' Hailong Xie, ${ }^{2}$ \\ Hongyu Deng,' Song Shang,' \\ Xiaohong Wang, ${ }^{4}$ Man Xia, ${ }^{5}$ \\ Chaohui Zuo' \\ 'Department of Gastroduodenal and \\ Pancreatic Surgery, Laboratory of Digestive \\ Oncology, Hunan Cancer Institute, \\ Hunan Cancer Hospital, Affiliated Cancer \\ Hospital of Xiangya School of Medicine, \\ Central South University, ${ }^{2}$ Institute of \\ Cancer Research, South China University, \\ ${ }^{3}$ Department of Laboratory Medicine, \\ Hunan Cancer Hospital, Affiliated Cancer \\ Hospital of Xiangya School of Medicine, \\ Central South University, ${ }^{4}$ Department of \\ Molecular Medicine, College of Biology, \\ State Key Laboratory of Chemo/Biosensing \\ and Chemometrics, Hunan University, \\ ${ }^{5}$ Department of Gynecological Oncology, \\ Hunan Cancer Hospital, Affiliated Cancer \\ Hospital of Xiangya School of Medicine, \\ Central South University, Changsha, \\ Hunan, China \\ *These authors contributed equally \\ to this work
}

Correspondence: Man Xia

Department of Gynecological Oncology, Hunan Cancer Hospital, Affiliated Cancer Hospital of Xiangya Medical School,

Central South University, 283 Tongzipo Road,

Changsha, Hunan 410013, China

Tel +86 I35 74I8 9795

Fax +8673189762142

Email xiamansummer@sina.com

Chaohui Zuo

Department of Gastroduodenal and Pancreatic Surgery, Laboratory of Digestive Oncology, Hunan Cancer Institute, Hunan Cancer Hospital, Affiliated Cancer Hospital of Xiangya Medical School, Central South University, 283 Tongzipo Road, Changsha,

Hunan 410013, China

Tel $+86 \quad$ I $38759 \mid \quad$ I 328

Fax +8673189762142

Email zuochaohui@vip.sina.com
Abstract: Worldwide, gastric cancer (GC) is one of the deadliest malignant tumors of the digestive system. Moreover, microRNAs (miRNAs) of exosomes harbored within cancer cells have been determined to induce inflammatory conditions that accelerate tumor growth and metastasis. Interestingly, the oncogenic role of bone marrow mesenchymal stem cells (BMMSCs) in the modulation of immunosuppression, tumor invasion, and metastasis was discovered to be partly mediated through the secretion of exosomes. In this article, high expression of miRNA-221 (miR-221) in exosomes of the peripheral blood was determined to be positively correlated with the poor clinical prognosis of GC, especially with respect to tumor, node, and metastases stage. Therefore, the expression of miR-221 in exosomes of the peripheral blood may be an important detection index for GC. Proliferation, migration, invasion, and adhesion to the matrix of GC BGC-823 and SGC-7901 cells were significantly enhanced by exosomes that originated from BM-MSCs that were transfected with miR-221 mimics. In conclusion, extracted exosomes from BM-MSCs transfected with miR-221 oligonucleotides can act as highefficiency nanocarriers, which can provide sufficient miR-221 oligonucleotides to influence the tumor microenvironment and tumor aggressiveness effectively. Notably, the use of a miR-221 inhibitor with an excellent restraining effect in exosomes provides therapeutic potential for GC in future clinical medicine.

Keywords: exosomes, miR-221, BM-MSCs, gastric cancer, prognosis, oncogenic activity

\section{Introduction}

Gastric cancer (GC) is one of the most common malignant neoplasms of the digestive system worldwide. A 2015 study reported 679,100 newly diagnosed GC cases in China, which account for $15.8 \%$ of the total number of newly diagnosed cancer patients. Worse still, 498,000 deaths occurred as a result of GC, and these accounted for $17.7 \%$ of all deaths from malignant tumors. ${ }^{1}$ Although the morbidity and mortality of GC have declined globally in recent years, this is not the case in China due to inadequate living conditions, dietary habits, and low efficiency of early diagnosis. ${ }^{2}$ Clinically, surgical resection is still the most effective therapeutic method for the treatment of GC. However, the prognosis of GC is not optimistic due to frequent metastasis to the lymph nodes and distant sites.

As a class of single-stranded RNAs that are 18-27 nucleotides in length, microRNAs (miRNAs) have been found to perform irreplaceable regulatory roles in many organisms. Some specific genes are modulated by miRNAs during the processes of RNA transcription, translation, and posttranslational modification. ${ }^{3}$ Therefore, research on miRNAs has become a hot topic worldwide, especially in cancer research. ${ }^{4,5}$ 
It was reported that miR-610 can suppress osteosarcoma oncogenesis via the targeting of TWIST1 expression. ${ }^{6}$ The proliferation and metastasis of stem cell-like cells in colorectal cancer are notably suppressed by miR-1280. ${ }^{7}$ These research findings indicate the significant regulatory roles of miRNAs in driving cancer initiation and development. Notably, the facilitating role of miRNA-221 (miR-221) in cancer cell proliferation, migration, and drug resistance has been widely recognized in recent years. For example, CD44 expression in hepatocellular carcinoma is regulated by miR-221 through the PI3K-AKT-mTOR pathway. ${ }^{8}$ Additionally, miR-221 can promote non-small-cell lung carcinoma by targeting TIMP $2 .{ }^{9}$ Hence, the expression of miR-221 may be a potential detection index in cancer, and miR-221 may serve as a therapeutic target.

In recent years, the increasingly important roles of bone marrow mesenchymal stem cells (BM-MSCs) in malignant neoplasms have garnered worldwide attention. Through modulation of the immune system and the secretion of some active factors used in the matrix formation of tumor cells, BM-MSCs participate in the constitution of the tumor microenvironment. ${ }^{10,11}$ Interestingly, the biological function of MSCs is performed through the secretion of small vesicles termed exosomes. ${ }^{12}$ As a type of membrane vesicle, exosomes are secreted by the exocytosis of multivesicular bodies. ${ }^{13-15}$ They are generally defined according to their spherical and unilamellar morphology, size (average diameter 30-100 nm), and the expression of specific biomarkers such as tetraspanins (CD9, CD63, and so on). ${ }^{16,17}$ Biological molecules that range from proteins and lipids to nucleic acids, including miRNA, mRNA, and other noncoding RNAs, are enriched in exosomes. Notably, the expression of miR-221 in exosomes was found to be frequently upregulated in GC-MSCs and some cancer tissues, and this increased expression is strongly associated with lymph node metastasis, venous invasion, and tumor, node, and metastases (TNM) stage. ${ }^{18,19}$ The regulatory role of miRNAs in exosomes has been determined to be an important mechanism in cancer development, ${ }^{20} \mathrm{drug}$ resistance, ${ }^{21}$ metastasis, ${ }^{22,23}$ and immune response. ${ }^{24}$

In our previous research, BM-MSCs were successfully isolated and identified as a favorable cell model for cancer research. ${ }^{25}$ Herein, miR-221 expression in exosomes from the peripheral blood was discovered to be highly associated with the TNM stage in GC. BM-MSC-derived exosomes that were transfected with miR-221 oligonucleotides were extracted for this study and were characterized by Brownian motion. The oncogenic roles of exosomal miR-221 in cell proliferation, migration, invasion, and adhesion to the matrix were repeatedly demonstrated through in vitro experiments. The extracted exosomes may possess adequate miR-221 oligonucleotides to further influence the biological properties of GC. miR-221 expression in exosomes may be an important tumor biomarker and may serve as a therapeutic target in the future.

\section{Materials and methods Cell culture}

Human BM-MSCs were isolated, identified, and preserved in our laboratory. ${ }^{25}$ The human GC cell line BGC-823 was purchased from Boster (Wuhan, China), and the SGC7901 cell line was kindly provided by Professor Hailong Xie from the University of South China. BM-MSCs were cultured in RPMI-1640 (Thermo Fisher Scientific, Waltham, MA, USA) supplemented with 15\% (v/v) fetal bovine serum (FBS; Thermo Fisher Scientific), 100 units/mL penicillin (Thermo Fisher Scientific), and $100 \mu \mathrm{g} / \mathrm{mL}$ streptomycin (Thermo Fisher Scientific). BGC-823 and SGC7901 cells were cultivated in Dulbecco's Modified Eagle's Medium (DMEM; Thermo Fisher Scientific) supplemented with $10 \%(\mathrm{v} / \mathrm{v}) \mathrm{FBS}$. The FBS used in cell culture was ultracentrifuged at $100,000 \times g$ for 16 hours to remove exosomes. All cells were cultured at $37^{\circ} \mathrm{C}$ in a humidified cell incubator with $5 \% \mathrm{CO}_{2}$.

\section{Isolation and characterization of exosomes}

The supernatants of cultured cells and peripheral blood were collected after centrifugation at $4^{\circ} \mathrm{C}$ for 5 minutes, and the exosomes were extracted using the ExoQuick-TCTM Exosome Precipitation Solution (System Biosciences, Palo Alto, CA, USA) according to the manufacturer's instructions. Briefly, supernatants were centrifuged at $10,000 \times g$ for 30 minutes to remove cell debris. The quick extraction solution EXOTC10A-1 (System Biosciences) was added to the filtered solution at a 1:5 ratio and was stored at $4{ }^{\circ} \mathrm{C}$ for at least 12 hours. Then, the mixture was centrifuged at $1,500 \times g$ for 30 minutes, and the exosomes precipitated at the bottom of the tubes. The extracted exosomes were dissolved in phosphate-buffered saline (PBS) and stored at $-80^{\circ} \mathrm{C}$. Protein in the exosomes was extracted using RIPA lysis buffer (Thermo Fisher Scientific), and protein expression of the typical markers CD9 and CD63 was examined by Western blotting. The size and concentration of exosomes were identified through an analysis of Brownian motion on a Microtrac S3500 analyzer (Microtrac, Montgomeryville, PA, USA). 


\section{Nanoparticle tracking analysis}

Exosomes derived from BM-MSCs were diluted with PBS at a 1:1,000 ratio, mixed so that a single particle was formed, and injected into the Microtrac S3500 instrument with a syringe to avoid small air bubbles. Brownian motion of exosomes was observed, and the size distribution and concentration of exosomes were recorded for further analysis.

\section{Transfection of miR-22I oligonucleotides}

The oligonucleotides of miR-221 mimics, miR-221 inhibitor, and negative control (NC) were synthesized and purified at Sangon Biotech (Shanghai, China). BM-MSCs were seeded in 6-well plates (Corning Incorporated, Corning, NY, USA) at $50 \%-60 \%$ confluence and were cultured overnight. The cells were then transfected with $100 \mathrm{nM}$ miRNA using Lipofectamine ${ }^{\circledR} 2000$ (Thermo Fisher Scientific) according to the manufacturer's instructions. After transfection for 48 hours, miR-221 expression was evaluated by quantitative real-time polymerase chain reaction (qRT-PCR). Table 1 lists the miRNA sequences.

\section{Exosome treatment in the GC cell lines BGC-823 and SGC-790I}

BGC-823 and SGC-7901 cells $\left(\sim 5 \times 10^{5}\right)$ were seeded in a 6-well culture plate at 50\%-60\% confluence. Then, $2 \mu \mathrm{g}$ exosomes (equivalent to those collected from $\sim 5 \times 10^{6} \mathrm{BM}-$ MSCs) per well, based on the protein concentration determined by a bicinchoninic assay (BCA; Sangon Biotech), were added to GC cells in the 6-well plate. After cultivation for 24 hours, exosomes were resuspended in PBS and added to the cells at a final concentration of $200 \mu \mathrm{g} / \mathrm{mL}$. The expression level of miR-221 in the cancer cells was analyzed 12 hours later by qRT-PCR.

\section{PCR}

Total RNA of cells was extracted using TRIzol (Thermo Fisher Scientific) and was treated with RNase-free DNase (Promega, Madison, WI, USA) at $37^{\circ} \mathrm{C}$ for 1 hour to remove genomic DNA. Reverse transcription-PCR of the RNA and qRT-PCR for specific genes were performed using

Table I Sequences of synthesized miR-22 I oligonucleotides

\begin{tabular}{ll}
\hline Oligonucleotides & Sequences $\left(5^{\prime}\right.$ to $\mathbf{3}^{\prime}$ ) \\
\hline miR-22I mimics & AGC UAC AUU GUC UGC UGG GUU UC \\
miR-22I inhibitor & GAA ACC CAG CAG ACA AUG UAG CU \\
Negative control & UUC UCC GAA CGU GUC ACG UdTdT \\
\hline
\end{tabular}

Abbreviation: miR-22I, microRNA-22I. the All-in-One ${ }^{\mathrm{TM}}$ miRNA qRT-PCR Detection System (GeneCopoeia, Rockville, MD, USA) according to the manufacturer's instructions. The specific primer for miR-221 was AGC TAC ATT GTC TGC TGG GTT TC. The U6 gene was used as the internal reference for qRT-PCR, the specific primer for which was purchased from GeneCopoeia.

From January 2016 to April 2016, patients with a pathological diagnosis of GC at Hunan Cancer Hospital and Affiliated Cancer Hospital of Xiangya Medical School were recruited for this study. Peripheral blood samples of $40 \mathrm{GC}$ patients and 20 normal controls were collected. Exosomes in the peripheral blood were extracted using the ExoQuick-TC ${ }^{\text {тм }}$ Exosome Precipitation Solution (System Biosciences) according to the manufacturer's instructions. Total RNA of exosomes in the peripheral blood was extracted using TRIzol. The expression level of miR-221 of exosomes was examined by qRT-PCR.

\section{Western blotting}

Cell lysates were obtained with RIPA lysis buffer (Thermo Fisher Scientific) on ice, were centrifuged at $4{ }^{\circ} \mathrm{C}$ for 15 minutes, and were quantified using a BCA kit. Proteins were run on a sodium dodecyl sulfate (SDS)-polyacrylamide gel electrophoresis gel according to a standard protocol and were transferred to a polyvinylidene difluoride membrane (Merck Millipore, Billerica, MA, USA). The following antibodies were used according to the manufacturer's instructions: CD9 (CST, Danvers, MA, USA), CD63 (CST), and $\beta$-actin (Sigma-Aldrich Co, St Louis, MO, USA). Specific protein bands were detected using the Chemiluminescent Substrate System (Thermo Fisher Scientific).

\section{MTT assay}

Cells in the logarithmic phase were seeded in a 96-well plate at a density of $1 \times 10^{4}$ cells per well. After cultivation at $37^{\circ} \mathrm{C}$ for 24 hours in a humidified incubator with $5 \% \mathrm{CO}_{2}$, the culture medium was changed to $100 \mu \mathrm{L}$ conditioned medium, which contained different types of exosomes. To determine the cell viability, $10 \mu \mathrm{L}$ of $5 \mathrm{mg} / \mathrm{mL}$ MTT solution (Sangon Biotech) was added to the culture medium and was incubated with the GC cells for 4 hours. Next, the culture medium was carefully removed, and $150 \mu \mathrm{L}$ dimethyl sulfoxide (Sangon Biotech) was added to each well. After shaking for 20 minutes, the optical density (OD) value at $490 \mathrm{~nm}$ was measured using a microplate reader (Thermo Fisher Scientific). The viability of the GC cells was tested 24 hours after they were seeded. All experiments were repeated three times. 


\section{Wound-healing assay}

Cells in the logarithmic phase were seeded in a 6-well plate at $50 \%-60 \%$ confluence. After culture for 24 hours, the cells underwent serum starvation in the culture medium without FBS for another 24 hours. The wound-healing assay was performed with a standard $10-\mu \mathrm{L}$ pipette tip and in cells that were $80 \%$ confluent. Then, the cells were further cultivated in serum-free medium for 24 hours. Images of the woundhealing process were captured every 12 hours using an inverted light microscope.

\section{Transwell assay}

GC cell invasion and migration experiments were conducted using Matrigel-coated and non-coated 24-well chambers with 8.0- $\mu \mathrm{m}$ PET membrane pores (Corning Incorporated), respectively. Briefly, $650 \mu \mathrm{L}$ culture medium with $10 \%$ FBS as the chemoattractant was added to the lower Transwell chamber. Then, $100 \mu \mathrm{L}$ serum-free DMEM with $1 \times 10^{5}$ cells was added to the upper chamber. After culture for 24 hours, the transmigrated cells were fixed in methyl alcohol, stained with $0.2 \%(\mathrm{~m} / \mathrm{v})$ crystal violet (Sigma-Aldrich $\mathrm{Co}$ ), and rinsed in PBS (Thermo Fisher Scientific). Images of the transmigrated cells were captured under an upright light microscope. Stained cells were further dissolved using $500 \mu \mathrm{L}$ of $1 \%$ mass/volume (m/v) SDS, and the number of transmigrated cells was qualitatively determined through the measurement of the OD value at $546 \mathrm{~nm}$.

\section{Adhesion assay}

A $96-w e l l$ plate was coated with $30 \mu \mathrm{L}$ of $40 \mu \mathrm{g} / \mathrm{mL}$ collagen I (Thermo Fisher Scientific) in PBS for 12 hours, then rinsed with PBS, and dried at room temperature. After serum starvation for 8 hours, the cells were detached after incubation with $10 \mathrm{mM}$ EDTA for 10 minutes, after which the cells were washed with DMEM to remove the EDTA, and resuspended at a density of $2 \times 10^{5}$ cells $/ \mathrm{mL}$ in DMEM with $0.1 \%(\mathrm{~m} / \mathrm{v})$ bovine serum albumin (TaKaRa, Tokyo, Japan). For the cell-substratum adhesion assay, $100 \mu \mathrm{L}$ of cell suspension was added to the coated wells. After incubation at $37^{\circ} \mathrm{C}$ for 20 minutes, the non-adherent cells were removed with $100 \mu \mathrm{L}$ DMEM in a total of four washes. The adherent cells were further cultured in DMEM supplemented with $10 \%$ (v/v) FBS at $37^{\circ} \mathrm{C}$ for 4 hours. The number of adherent cells was determined by MTT assays.

\section{Ethics approval}

This study was approved by the Institutional Review Board of Hunan Cancer Hospital affiliated with Xiangya Medical
School. All human tissue samples were obtained with written informed consent and were approved for use by the Ethics Committee of Hunan Cancer Hospital and the Affiliated Cancer Hospital of Xiangya Medical School.

\section{Statistical analysis}

SPSS 19.0 statistical software (IBM Corporation, Armonk, NY, USA) was used for all statistical analyses. Student's $t$-test and one-way analysis of variance were used in the statistical analysis. Graphs were generated using GraphPad Prism 5.0 (GraphPad Software, La Jolla, CA, USA). Results were expressed as the mean \pm standard deviation. Differences were considered statistically significant at $*(P<0.05)$, $* *(P<0.01)$, and $* * *(P<0.001)$.

\section{Results \\ miR-22I expression level in exosomes from peripheral blood is positively correlated with poor clinical prognosis of GC}

From January 2016 to April 2016, exosomes in peripheral blood samples of $40 \mathrm{GC}$ patients and 20 normal controls were extracted for this study. The specific expression of CD9 and CD63 in the exosomal lysate of representative samples indicated the successful isolation of exosomes from peripheral blood (Figure 1A). RNA present in the extracted exosomes was extracted to analyze the expression of miR-221. According to the results of qRT-PCR assays, the miR-221 expression level in exosomes from the peripheral blood of GC patients was significantly higher (2.5-fold) than that in the peripheral blood of controls (Figure 1B). Therefore, the miR-221 expression in exosomes from the peripheral blood was significantly upregulated in GC. Although no significant difference was observed with respect to gender, age, tumor location, and tumor size, the expression level of miR-221 was closely associated with the TNM stage of GC in clinical samples (Table 2). Therefore, miR-221 expression in exosomes of the peripheral blood may have some significant clinical value. According to these results, we speculated that the upregulated expression of exosomal miR-221 may play an important role in the proliferation, migration, and invasiveness of GC cells.

\section{Characteristics of exosomes}

To verify our findings, BM-MSCs were transfected with different miR-221 oligonucleotides, as shown in Table 1. After transfection for 48 hours, exosomes from BM-MSCs were 

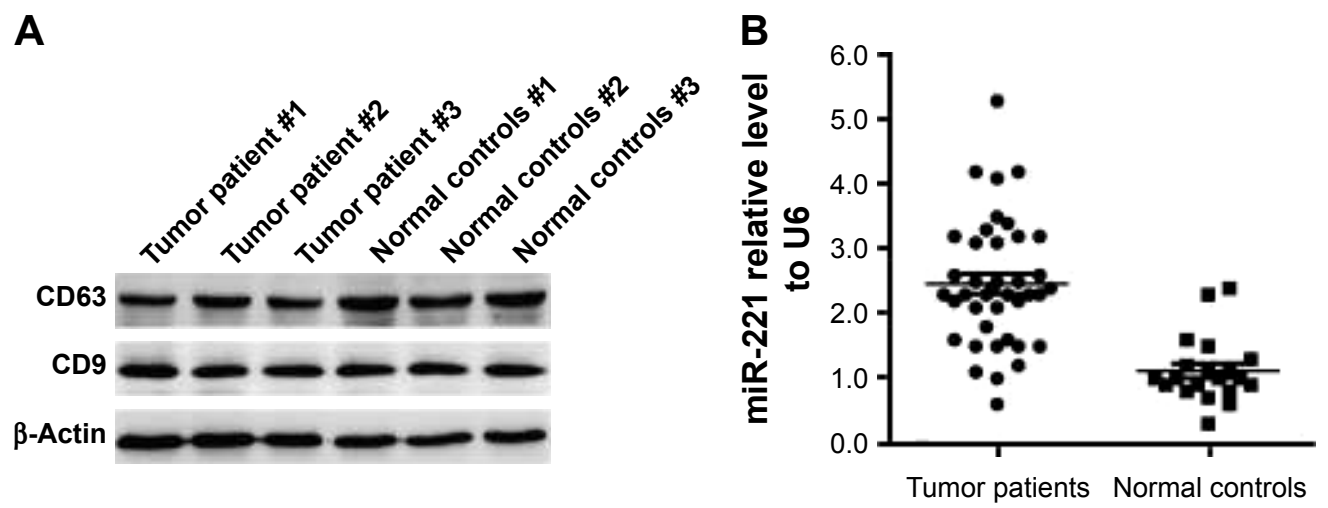

Figure I The expression of miR-22I in exosomes of the peripheral blood is closely associated with the prognosis of GC.

Notes: (A) The expression of the biomarkers CD9 and CD63, as examined by Western blotting, indicated the successful extraction of exosomes from the peripheral blood. $\beta$-actin was used as the internal reference. (B) From January 2016 to April 2016, peripheral blood samples were obtained from $40 \mathrm{GC}$ patients and 20 normal controls who were recruited for this research. miR-22I expression in exosomes of the peripheral blood of GC patients, as assessed by qRT-PCR, was generally higher than that of the normal controls. U6 was used as the internal control.

Abbreviations: miR-22I, microRNA-22I; GC, gastric cancer; qRT-PCR, quantitative real-time polymerase chain reaction.

extracted for further study. As they are biological markers of exosomes, CD9 and CD63 protein expression was tested by Western blotting, which indicated successful extraction of exosomes of BM-MSCs (Figure 2A). According to the analytical results of Brownian motion, the diameter spread of exosomes was basically consistent with normal distribution (Figure 2B). Specifically, the diameter of the exosomes primarily ranged from 20 to $120 \mathrm{~nm}$, and the concentration of exosomes with a diameter of $71 \mathrm{~nm}$ was observed to have a peak value. After transfection with miR-221 mimics and an inhibitor for 48 hours, the miR-221 expression level was correspondingly changed in BM-MSCs (Figure 2C). Compared with the $\mathrm{NC}$ group, the expression of miR-211 in exosomes was significantly upregulated over 150 -fold after transfection with miR-221 mimics. Moreover, the expression of miR-211 in the inhibitor group was critically inhibited by $\sim 99 \%$ compared with the expression in the NC group. These results indicated that miR-221 expression can be effectively modulated through in vitro transfection.

\section{miR-22I of exosomes extracted from BM-MSCs can accelerate GC cell proliferation}

To evaluate the role of miR-221 in exosomes in the regulation of cancer properties, the extracted exosomes were added to the culture medium of the GC cells BGC-823 and SGC-7901. After they were cultured for 12 hours, miR-221 expression in GC cells was determined by qRT-PCR. Compared with that of the $\mathrm{NC}$ group, the expression of miR-221 in BGC-823 and SGC-7901 cells increased 15- and 13-fold, respectively, in the group transfected with mimics, while the expression of miR-221 was reduced $15 \%$ and $10 \%$ in BGC823 and SGC-7901 cells, respectively, that were transfected with the inhibitor (Figure 3A). These results indicated that the extracted exosomes may contain sufficient transfected miR-221 oligonucleotides, which can enter GC cells and further affect miR-221 expression. Similar to liposome-mediated transfection, exosomes may act as nanocarriers to influence gene expression. To confirm the efficiency of exosome transfection, the protein expression of PTEN and p27, which are direct targets regulated by miR-221, ${ }^{19,26,27}$ was examined by Western blotting. Compared with the NC group, the expression levels of PTEN and p27 were downregulated by oligonucleotides of miR-221 mimics, while their expression levels were upregulated by oligonucleotides of the miR-221 inhibitor (Figure 3B); this suggests the high efficiency of exosome transfection. Moreover, the proliferation of BGC-823 and SGC-7901 cells as examined by MTT was enhanced after culture with miR-221 mimics (Figure 3C). In comparison, cell proliferation was attenuated after treatment with the miR221 inhibitor. In conclusion, the transfected exosomes may act as natural nanocarriers that contain sufficient miR-221 oligonucleotides that can further influence GC cell proliferation. These results also indicated the therapeutic potential of exosomal miR-221 in future clinical medicine.

\section{miR-22I in exosomes extracted from BM-MSCs effectively promotes GC cell migration, invasion, and adhesion to the matrix}

Wound-healing assays were conducted to evaluate the effect of miR-221 in the regulation of GC cell migration. 
Table 2 Correlation of GC clinicopathologic features and miR221 expression of exosomes in peripheral blood $(n=40)$

\begin{tabular}{|c|c|c|c|}
\hline Parameters & Cases & $\begin{array}{l}\text { Relative level } \\
\text { to U6 }\end{array}$ & $P$-value \\
\hline Sex & & & 0.80 \\
\hline Male & 22 & $2.46(0.60-4.20)$ & \\
\hline Female & 18 & $2.48(1.10-5.30)$ & \\
\hline Age & & & 0.47 \\
\hline$<60$ & 19 & $2.22(1.00-4.20)$ & \\
\hline$\geq 60$ & 21 & $2.69(0.60-5.30)$ & \\
\hline CEA grading & & & 0.46 \\
\hline Normal & 18 & $2.47(0.60-4.20)$ & \\
\hline High & 22 & $2.46(1.10-5.30)$ & \\
\hline CA19-9 grading & & & 0.73 \\
\hline Normal & 31 & $2.4 \mathrm{I}(0.60-5.30)$ & \\
\hline High & 9 & $2.67(1.10-4.20)$ & \\
\hline CRP grading & & & 0.49 \\
\hline Normal & 25 & $2.37(0.60-5.30)$ & \\
\hline High & 15 & $2.62(1.50-4.20)$ & \\
\hline Tumor location & & & 0.25 \\
\hline Gastric fundus and cardiac & 7 & $2.32(1.50-4.20)$ & \\
\hline Gastric body & 11 & $2.26(1.10-4.10)$ & \\
\hline Gastric antrum & 22 & $2.39(0.60-5.30)$ & \\
\hline Tumor size & & & 0.59 \\
\hline$<4.0 \mathrm{~cm}$ & 20 & $2.57(0.60-5.30)$ & \\
\hline$\geq 4.0 \mathrm{~cm}$ & 20 & $2.37(1.00-4.20)$ & \\
\hline Lauren type & & & 0.94 \\
\hline Intestinal type & 19 & $2.44(1.10-4.20)$ & \\
\hline Diffuse type & 9 & $2.41(1.50-4.10)$ & \\
\hline Mixed type & 12 & $2.55(0.60-5.30)$ & \\
\hline Borrmann type & & & 0.82 \\
\hline I & 3 & $2.63(1.50-4.10)$ & \\
\hline$\|$ & 17 & $2.35(0.60-5.30)$ & \\
\hline III & 20 & $2.54(1.00-4.20)$ & \\
\hline \multicolumn{2}{|c|}{ Presence of vascular and nerve invasion } & & 0.16 \\
\hline No & 16 & $2.14(1.10-3.40)$ & \\
\hline Yes & 24 & $2.68(0.60-5.30)$ & \\
\hline Histological grade & & & 0.48 \\
\hline Well differentiated & 2 & $3.25(3.20-3.30)$ & \\
\hline Moderately differentiated & 6 & $2.33(1.00-4.20)$ & \\
\hline Poorly differentiated & 28 & $2.37(0.60-4.20)$ & \\
\hline Undifferentiated & 4 & $2.93(1.50-5.30)$ & \\
\hline T staging & & & 0.83 \\
\hline TI & 5 & $2.20(1.50-2.60)$ & \\
\hline $\mathrm{T} 2$ & 11 & $2.36(0.60-5.30)$ & \\
\hline T3 & 21 & $2.60(1.10-4.20)$ & \\
\hline T4 & 3 & $2.30(2.20-2.40)$ & \\
\hline $\mathrm{N}$ staging & & & 0.32 \\
\hline No & 8 & $2.01(0.60-2.60)$ & \\
\hline NI & 8 & $2.34(1.10-3.30)$ & \\
\hline N2 & 13 & $2.50(1.00-4.20)$ & \\
\hline N3 & 11 & $2.85(1.50-5.30)$ & \\
\hline M staging & & & 0.15 \\
\hline Mo & 38 & $2.37(0.60-5.30)$ & \\
\hline MI & 2 & $4.20(4.20,4.20)$ & \\
\hline TNM stage & & & 0.02 \\
\hline I & 5 & I. $82(0.60-2.60)$ & \\
\hline II & 15 & $2.33(1.00-4.20)$ & \\
\hline III & 18 & $2.56(1.20-5.30)$ & \\
\hline IV & 2 & $4.20(4.20,4.20)$ & \\
\hline
\end{tabular}

Abbreviations: TNM, tumor, node, and metastasis; miR-22I, microRNA-22I; GC, gastric cancer; CRP, C-reactive protein; CEA, carcinoembryonic antigen.
Although no obvious difference was observed at 12 hours, the rate of wound healing in BGC-823 and SGC-7901 cells was accelerated in the miR-221 mimics groups at 24 hours (Figure 4A and B). In comparison, the rate of wound healing was attenuated in the inhibitor groups. These results were further validated by Transwell assays. Compared with that of the NC groups, the number of BGC-823 and SGC-7901 cells that had transmigrated was significantly increased 2.23- and 1.75-fold, respectively, after culture with exosomes that were transfected with miR-221 mimics. However, the number of BGC-823 and SGC-7901 cells that had transmigrated was decreased 0.6- and 0.8-fold, respectively, after culture with exosomes transfected with an inhibitor (Figure 4C and D). These results indicated that miR-221 of exosomes can effectively promote GC cell migration.

The role of exosomal miR-221 in the regulation of cell invasion was evaluated using coated Transwell chambers. It is clear that the invasion ability of BGC-823 and SGC7901 cells was significantly upregulated after culture with exosomes transfected with miR-221 mimics (Figure 4E). In contrast, the invasion ability of GC cells was significantly suppressed after culture with exosomes transfected with the miR-221 inhibitor. These results indicated that the extracted exosomes from transfected BM-MSCs can further influence the invasion ability of GC cells and that miR-221 in exosomes promotes the invasiveness of GC cells.

The assessment of the ability of exosomal miR-221 to regulate adhesion of $\mathrm{GC}$ cells to the matrix was conducted using adhesion assays. Consequently, threefold of BGC-823 cells cultured with exosomes that were treated with miR-221 mimics successfully adhered to the matrix compared with the number in the NC group (Figure 4F). Similar results were observed in SGC-7901 cells. In contrast, the number of adherent GC cells was significantly decreased after culture with exosomes transfected with miR-221 inhibitor. In conclusion, exosomes with high expression of miR-221 can effectively promote GC cell migration, invasion, and adhesion to the matrix. Therefore, a sufficient amount of miR-221 mimics and inhibitor existed in exosomes and effectively contributed to the oncogenic activity of GC cells. These upregulated biological properties of GC cells triggered by miR-221 mimics transfected into exosomes may be a new mechanism that explains the frequent metastasis of GC. According to these results, it is not difficult to understand why the high expression of exosomal miR-221 in peripheral blood is positively correlated with the poor clinical prognosis of GC. 
A

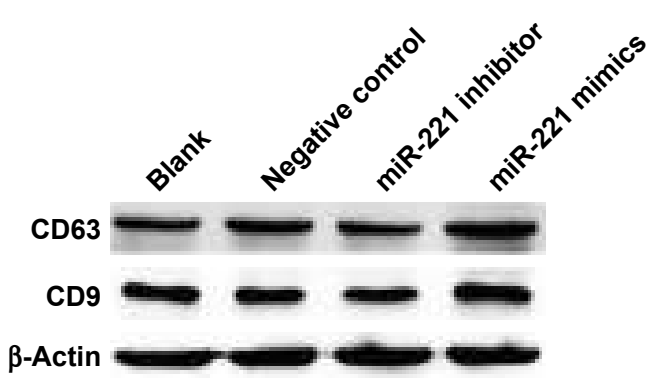

C

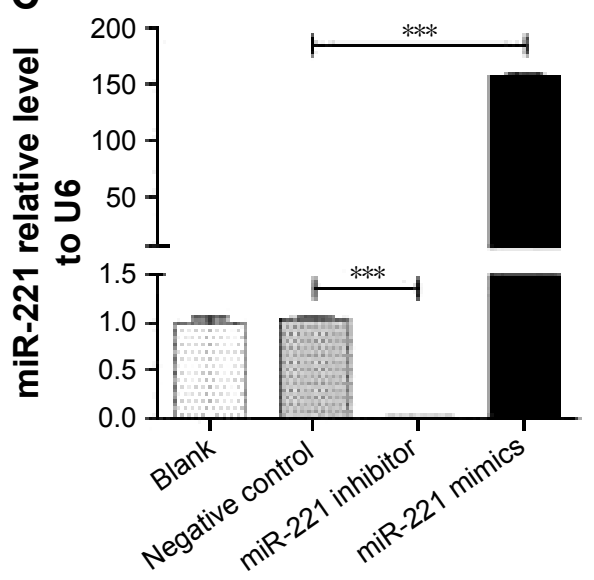

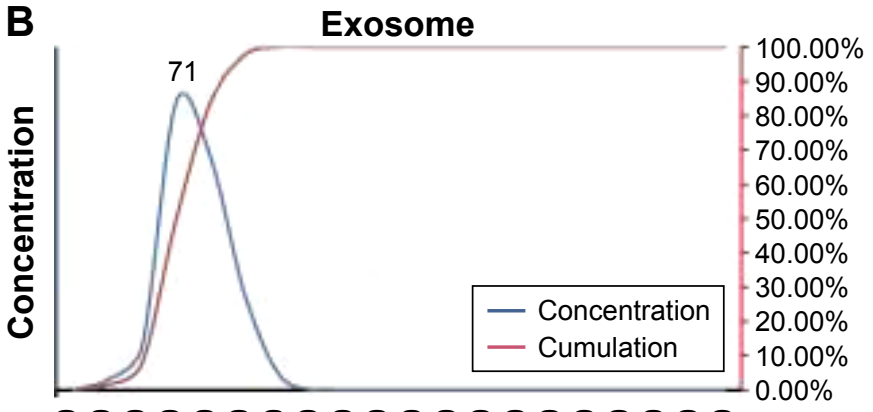

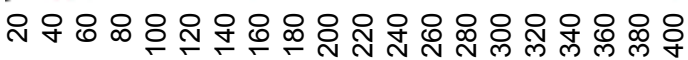
Particle size (nm)

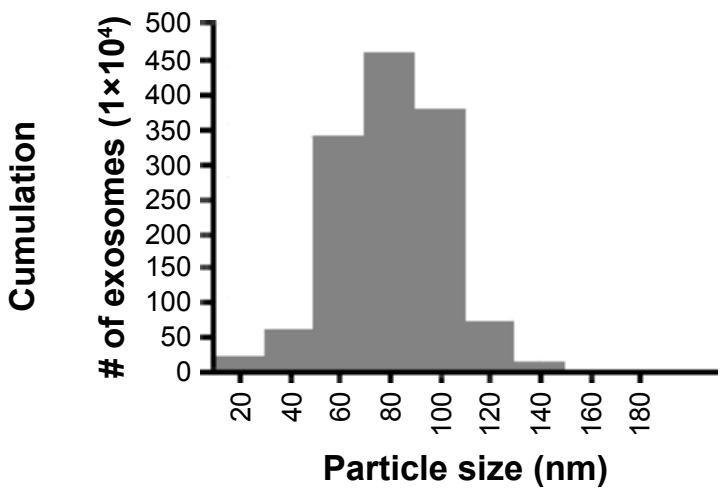

Figure 2 Characterization of exosomes that originated from BM-MSCs.

Notes: (A) Protein expression of CD9 and CD63 according to Western blotting indicated the successful extraction of exosomes from BM-MSCs. $\beta$-actin was used as the internal reference. (B) The particle size $(\mathrm{nm})$ of exosomes from BM-MSCs after transfection with miR-22I mimics was assayed by nanoparticle tracking analysis. (C) After transfection with miR-22I mimics or inhibitor for 48 hours, the miR-22I expression level was assessed by qRT-PCR and was correspondingly changed in BM-MSCs. U6 was used as the internal control. The difference was considered statistically significant at $* * *(P<0.00 \mathrm{I})$.

Abbreviations: BM-MSCs, bone marrow mesenchymal stem cells; miR-22I, microRNA-22I; qRT-PCR, quantitative real-time polymerase chain reaction.

\section{Discussion}

As clinically relevant cells, MSCs (especially BM-MSCs) are believed to be responsible for constituting an immunosuppressive microenvironment and a mesenchymal niche for the maintenance of cancer stem cells, tumor growth, angiogenesis, and metastasis. However, some specific MSCs also play a role in cancer resistance. For example, MSCs derived from adipose tissue and umbilical cord/cord blood can inhibit blood, breast, and digestive system cancer cell proliferation and tumor growth in vitro and in vivo. ${ }^{3,28}$ Therefore, recognition of the biological properties of MSCs may help us to develop more effective methods for cancer therapy.

As a subgroup of extracellular vehicles, exosomes were found to mediate long distance intercellular communication without direct cell-to-cell contact. ${ }^{29,30}$ Combined with the absolute predominance in biocompatibility, exosomes are considered to be natural nanocarriers that can be used in clinical applications, such as drug delivery. ${ }^{29,30}$ Some specific mRNAs, regulatory miRNAs, lipids, and proteins in exosomes each perform distinctly important roles in the promotion of cancer epithelial-mesenchymal transition and drug resistance. ${ }^{31,32}$ Notably, some oncogenic miRNAs, which are harbored in exosomes that originate from cancer cells, have been found to induce inflammatory conditions that accelerate tumor growth and metastasis. ${ }^{33}$ For example, exosomal miR-221 can induce tumor progression and temozolomide resistance in glioma by targeting DNM3. ${ }^{34}$ The exosome-mediated transfer of miR-222 increases the malignancy of melanoma. ${ }^{35}$ These findings provide future research directions with respect to miRNAs and exosomebased diagnostics and therapies for malignant neoplasms.

In this article, a high expression level of miR-221 in exosomes from the peripheral blood was determined to have positive correlation with the poor clinical prognosis of GC. It appears that miR-221 in exosomes plays a critical role in GC development and metastasis, and may serve as a 
A

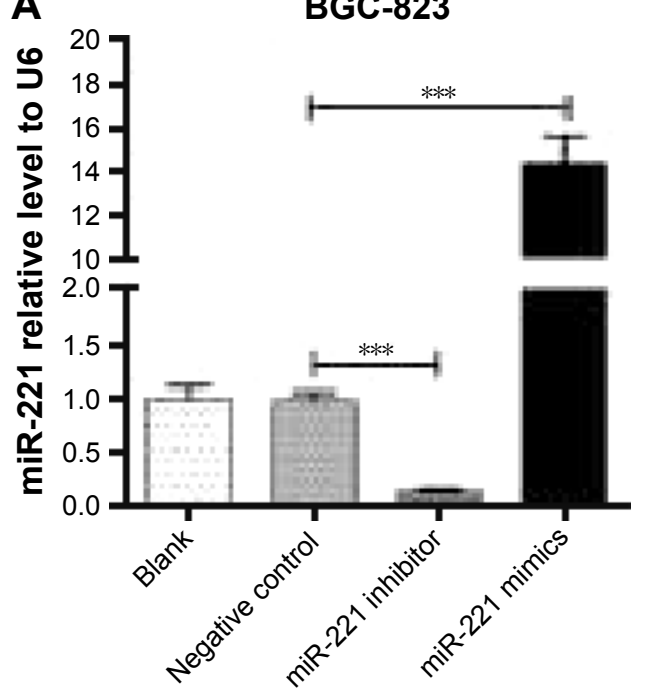

B

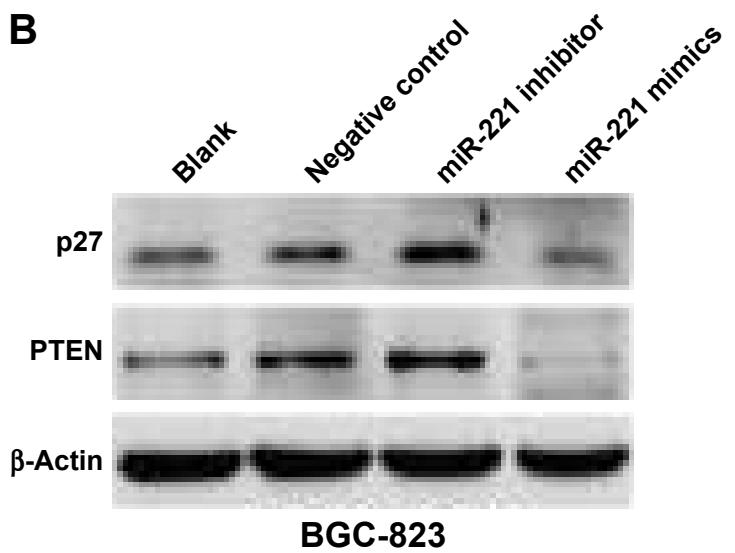

C

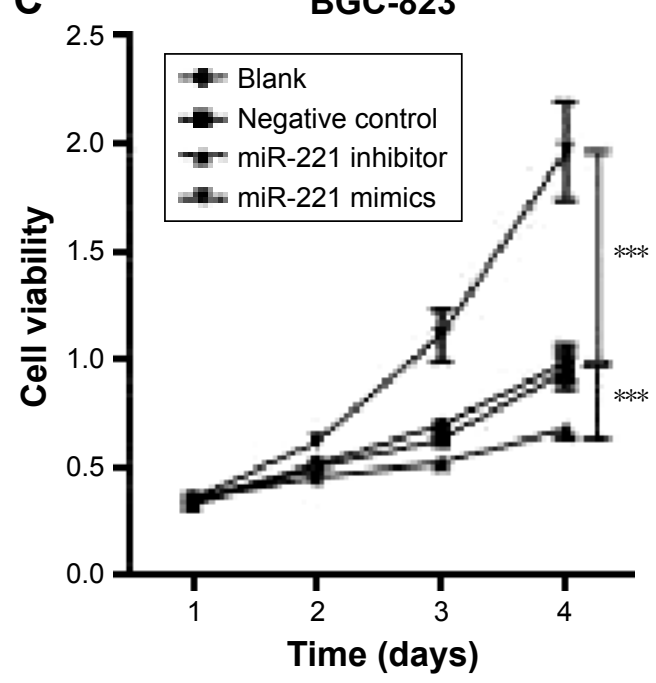

SGC-7901
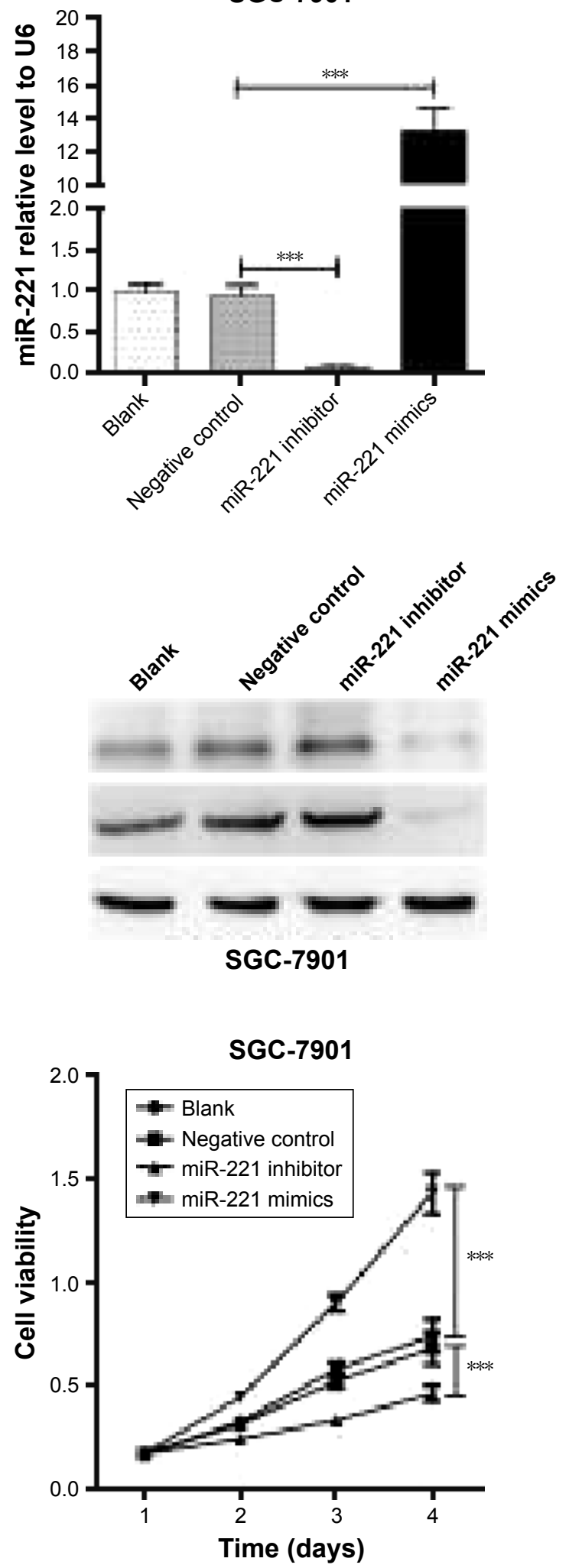

Figure 3 miR-22I in exosomes that originated from BM-MSCs promotes GC cell proliferation.

Notes: (A) miR-22I expression in BGC-823 and SGC-790I cells, which was determined by qRT-PCR, was increased in the mimics group but reduced in the inhibitor group. U6 was used as the internal reference. (B) The corresponding expression of PTEN and p27, as examined by Western blotting in GC cells cultured with exosomes, suggested the feasibility of exosome transfection. (C) Viability of BGC-823 and SGC-790 I cells, as determined by MTT, was significantly enhanced in the miR-22I mimics group compared with the miR-22I inhibitor group. The difference was considered statistically significant at $* * *(P<0.00 \mathrm{I})$.

Abbreviations: miR-22I, microRNA-22I; BM-MSCs, bone marrow mesenchymal stem cells; GC, gastric cancer; qRT-PCR, quantitative real-time polymerase chain reaction. 
A
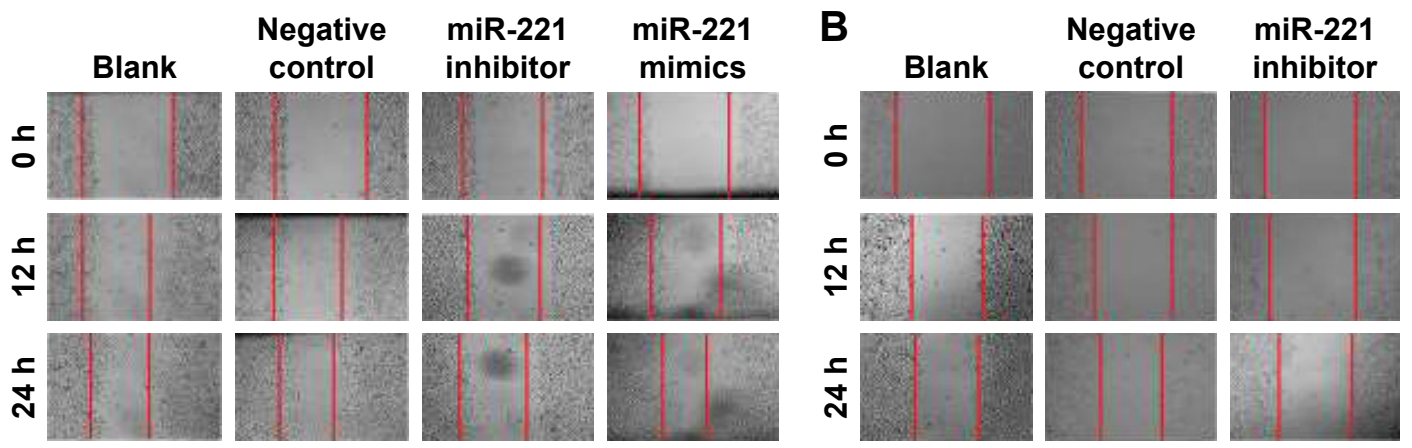

miR-221
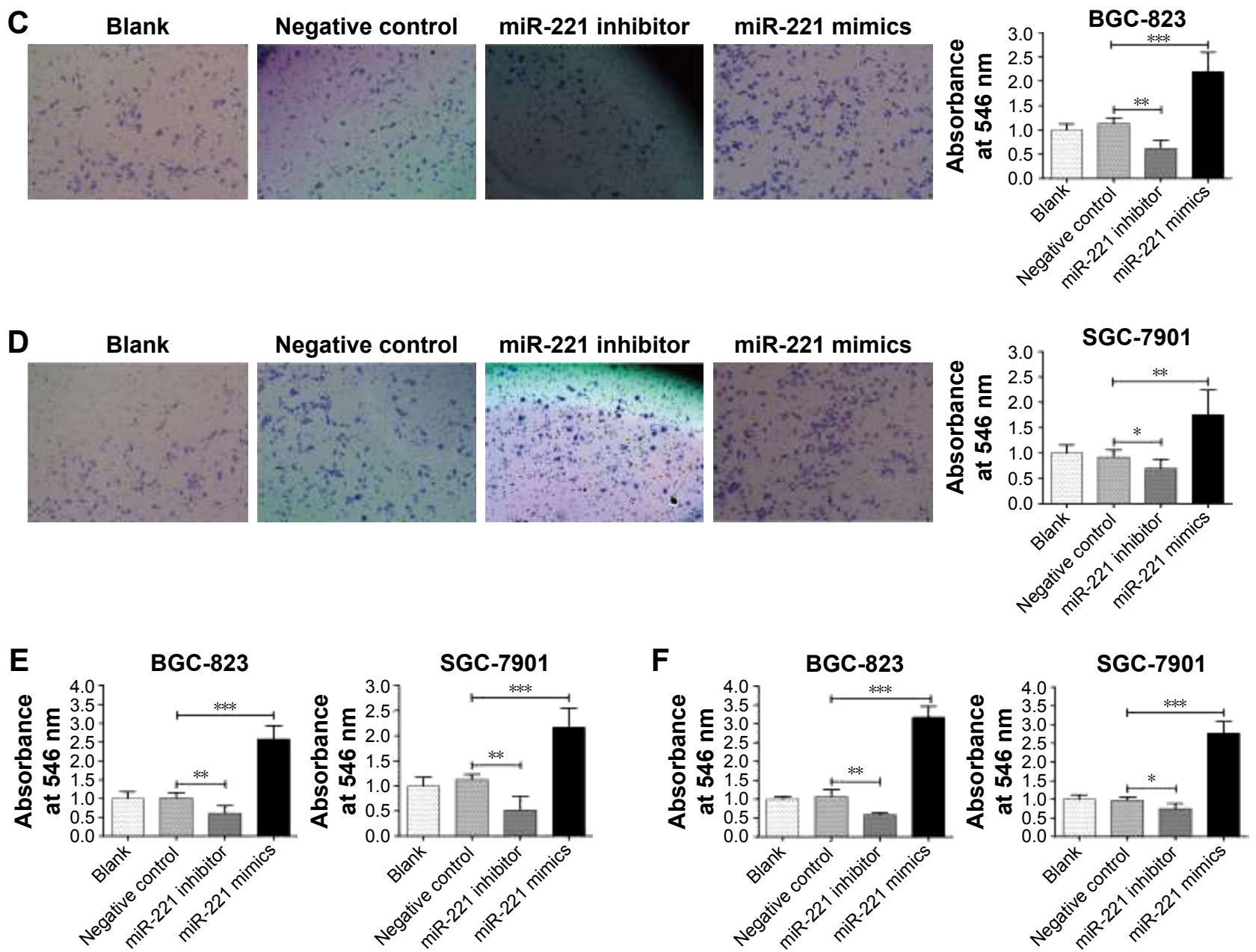

Figure 4 miR-22I in exosomes that originated from BM-MSCs promotes GC cell migration, invasion, and adhesion to the matrix.

Notes: (A-D) GC cell migration ability, which was evaluated by wound-healing and Transwell assays, was enhanced after culture with exosomes transfected with miR-22I mimics, while cell migration was restrained in cells cultured with exosomes that were transfected with miR-22I inhibitor. Histograms show the OD value of the BGC-823 and SGC-790I cells that transmigrated in the Transwell assays and that were stained with crystal violet. (E) GC cell invasion ability, which was evaluated by Transwell assays, was upregulated after culture with exosomes transfected with miR-22I mimics, while invasion ability was downregulated in cells cultured with exosomes transfected with miR-22I inhibitor. Histograms show the OD value of the invasive BGC-823 and SGC-790I cells that were stained with crystal violet. (F) Adhesion to the matrix of GC cells, as tested by adhesion assays, was significantly higher in the cells cultured with exosomes transfected with miR-22I mimics than in the cells cultured with exosomes transfected with the miR-22I inhibitor. Histograms show the OD value of adherent BGC-823 and SGC-790I cells, as shown by MTT assay. The difference was considered statistically significant at $*(P<0.05)$, $* *(P<0.0 \mathrm{I})$, and $* * *(P<0.00 \mathrm{I})$.

Abbreviations: miR-22I, microRNA-22I; BM-MSCs, bone marrow mesenchymal stem cells; GC, gastric cancer; OD, optical density.

detection index for neoplasms. The extracted exosomes with different miR-221 expression levels were identified to have significant regulatory roles in GC cell proliferation, migration, invasion, and adhesion. These results led us to further research in which we revealed the detailed mechanism of the function of miR-221 in exosomes. Our research also indicated that, through the in vitro transfection of exosomes, sufficient miR-221 oligonucleotides can exist in exosomes, 
which can further transform the biological characteristics of GC cells. The remarkable effect of exosomes treated with miR-221 inhibitor provides evidence for the potential use of this approach for gene therapy in GC. Additional experiments will be performed to reveal the detailed mechanisms of exosomal miR-221 in the promotion of GC development and metastasis.

Considering the moderate developments in the detection of markers in the blood, miRNAs in the peripheral blood will be useful as diagnostic markers. ${ }^{36-38}$ Moreover, exosomes secreted from cancer lesions may contribute to the formation of the tumor microenvironment, which facilitates frequent metastasis to lymph nodes and distant sites. Hence, miR-221 expression in exosomes from the peripheral blood may better reflect the potential risk for GC and other malignant neoplasms in certain individuals. The timely detection of exosomal miR-221 may be very valuable for the early diagnosis of GC. In addition, exosomes in the peripheral blood can be developed as nanocarriers that regulate the in vivo expression of miR-221. The controlled expression of miR-221 in exosomes of the peripheral blood may be helpful for the mitigation and treatment of GC in the future.

\section{Acknowledgments}

This research was supported by the Hunan Province Health Department of China (grant no B2013101, to Professor Chaohui Zuo), the Hunan Province Natural Science Foundation of China (grant no 2015JJ6063, to Professor Chaohui Zuo), and the National Natural Science Foundation of China (grant no 81500150, to Dr Man Xia).

\section{Disclosure}

Professor Chaohui Zuo and Professor Man Xia are married. The authors report no other conflicts of interest in this work.

\section{References}

1. Chen $\mathrm{W}$, Zheng R, Baade PD, et al. Cancer statistics in China, 2015. CA Cancer J Clin. 2016;66(2):115-132.

2. Siegel RL, Miller KD, Jemal A. Cancer statistics, 2017. CA Cancer J Clin. 2017;67(1):7-30.

3. Zhang C, Yang S-J, Wen Q, et al. Human-derived normal mesenchymal stem/stromal cells in anticancer therapies. J Cancer. 2017;8(1):85-96.

4. Cochetti G, Poli G, Guelfi G, Boni A, Egidi MG, Mearini E. Different levels of serum microRNAs in prostate cancer and benign prostatic hyperplasia: evaluation of potential diagnostic and prognostic role. Onco Targets Ther. 2016;9:7545-7553.

5. Chen MW, Yang ST, Chien MH, et al. The STAT3-miRNA-92-Wnt signaling pathway regulates spheroid formation and malignant progression in ovarian cancer. Cancer Res. 2017;77(8):1955-1967.

6. Jin C, Feng Y, Ni Y, Shan Z. MicroRNA-610 suppresses osteosarcoma oncogenicity via targeting TWIST1 expression. Oncotarget. Epub 2017 Apr 11.
7. Huang B, Yang H, Cheng X, et al. tRF/miR-1280 suppresses stem cell-like cells and metastasis in colorectal cancer. Cancer Res. 2017; 77(12):3194-3206.

8. Kim J, Jiang J, Badawi M, Schmittgen TD. miR-221 regulates CD44 in hepatocellular carcinoma through the PI3K-AKT-mTOR pathway. Biochem Biophys Res Commun. 2017;487(3):709-715.

9. Yin Z, Xu M, Li P. miRNA-221 acts as an oncogenic role by directly targeting TIMP2 in non-small-cell lung carcinoma. Gene. 2017;620:46-53.

10. Foronjy RF, Majka SM. The potential for resident lung mesenchymal stem cells to promote functional tissue regeneration: understanding microenvironmental cues. Cells. 2012;1(4):874.

11. Luo J, Ok Lee S, Liang L, et al. Infiltrating bone marrow mesenchymal stem cells increase prostate cancer stem cell population and metastatic ability via secreting cytokines to suppress androgen receptor signaling. Oncogene. 2014;33(21):2768-2778.

12. Ono M, Kosaka N, Tominaga N, et al. Exosomes from bone marrow mesenchymal stem cells contain a microRNA that promotes dormancy in metastatic breast cancer cells. Sci Signal. 2014;7(332):ra63.

13. Mazzeo C, Calvo V, Alonso R, Merida I, Izquierdo M. Protein kinase $\mathrm{D} 1 / 2$ is involved in the maturation of multivesicular bodies and secretion of exosomes in T and B lymphocytes. Cell Death Differ. 2016; 23(1):99-109.

14. Ceccarelli S, Visco V, Raffa S, Wakisaka N, Pagano JS, Torrisi MR. Epstein-Barr virus latent membrane protein 1 promotes concentration in multivesicular bodies of fibroblast growth factor 2 and its release through exosomes. Int J Cancer. 2007;121(7):1494-1506.

15. Heijnen HF, Schiel AE, Fijnheer R, Geuze HJ, Sixma JJ. Activated platelets release two types of membrane vesicles: microvesicles by surface shedding and exosomes derived from exocytosis of multivesicular bodies and alpha-granules. Blood. 1999;94(11):3791-3799.

16. Lee C, Carney RP, Hazari S, et al. 3D plasmonic nanobowl platform for the study of exosomes in solution. Nanoscale. 2015;7(20):9290-9297.

17. Rana S, Malinowska K, Zoller M. Exosomal tumor microRNA modulates premetastatic organ cells. Neoplasia. 2013;15(3):281-295.

18. Wang M, Zhao C, Shi H, et al. Deregulated microRNAs in gastric cancer tissue-derived mesenchymal stem cells: novel biomarkers and a mechanism for gastric cancer. Br J Cancer. 2014;110(5):1199-1210.

19. Matsuzaki J, Suzuki H. Role of microRNAs-221/222 in digestive systems. J Clin Med. 2015;4(8):1566-1577.

20. Xie SY, Li YJ, Wang PY, Jiao F, Zhang S, Zhang WJ. miRNA-regulated expression of oncogenes and tumor suppressor genes in the cisplatininhibited growth of K562 cells. Oncol Rep. 2010;23(6):1693-1700.

21. Niu J, Xue A, Chi Y, et al. Induction of miRNA-181a by genotoxic treatments promotes chemotherapeutic resistance and metastasis in breast cancer. Oncogene. 2016;35(10):1302-1313.

22. Wang T, Xu H, Qi M, Yan S, Tian X. miRNA dysregulation and the risk of metastasis and invasion in papillary thyroid cancer: a systematic review and meta-analysis. Oncotarget. Epub 2017 Mar 29.

23. Rokavec M, Horst D, Hermeking H. Cellular model of colon cancer progression reveals signatures of mRNAs, miRNA, IncRNAs, and epigenetic modifications associated with metastasis. Cancer Res. 2017;77(8):1854-1867.

24. Zhou R, Hu G, Liu J, Gong AY, Drescher KM, Chen XM. NF-kappaB p65-dependent transactivation of miRNA genes following Cryptosporidium parvum infection stimulates epithelial cell immune responses. PLoS Pathog. 2009;5(12):e1000681.

25. Zhou K, Xia M, Tang B, et al. Isolation and comparison of mesenchymal stem cell-like cells derived from human gastric cancer tissues and corresponding ovarian metastases. Mol Med Rep. 2016;13(2): 1788-1794

26. Chiba M, Kimura M, Asari S. Exosomes secreted from human colorectal cancer cell lines contain mRNAs, microRNAs and natural antisense RNAs, that can transfer into the human hepatoma HepG2 and lung cancer A549 cell lines. Oncol Rep. 2012;28(5):1551-1558.

27. Qin G, Yu B, Gong M, et al. Cardiomyocyte protection by GATA-4 gene engineered mesenchymal stem cells is partially mediated by translocation of miR-221 in microvesicles. PLoS One. 2013;8(8):e73304. 
28. Gao L, Zhang C, Zhang X, Gao L, Hao L, Chen XH. Human umbilical cord blood-derived stromal cells: a new resource for the proliferation and apoptosis of myeloma cells. Hematology. 2014;19(3):148-157.

29. Syn NL, Wang L, Chow EK, Lim CT, Goh BC. Exosomes in cancer nanomedicine and immunotherapy: prospects and challenges. Trends Biotechnol. 2017;35(7):665-676.

30. Luan X, Sansanaphongpricha K, Myers I, Chen H, Yuan H, Sun D. Engineering exosomes as refined biological nanoplatforms for drug delivery. Acta Pharmacol Sin. 2017;38(6):754-763.

31. Qu L, Ding J, Chen C, et al. Exosome-transmitted lncARSR promotes sunitinib resistance in renal cancer by acting as a competing endogenous RNA. Cancer Cell. 2016;29(5):653-668.

32. Weidle UH, Birzele F, Kollmorgen G, Ruger R. The multiple roles of exosomes in metastasis. Cancer Genomics Proteomics. 2017;14(1):1-15.

33. Melo SA, Sugimoto H, O’Connell JT, et al. Cancer exosomes perform cell-independent microRNA biogenesis and promote tumorigenesis. Cancer Cell. 2014;26(5):707-721.
34. Yang JK, Yang JP, Tong J, et al. Exosomal miR-221 targets DNM3 to induce tumor progression and temozolomide resistance in glioma. J Neurooncol. 2017;131(2):255-265.

35. Felicetti F, De Feo A, Coscia C, et al. Exosome-mediated transfer of miR-222 is sufficient to increase tumor malignancy in melanoma. J Transl Med. 2016;14:56.

36. Zeng RC, Zhang W, Yan XQ, et al. Down-regulation of miRNA-30a in human plasma is a novel marker for breast cancer. Med Oncol. 2013; 30(1):477

37. Weng Y, Chen Y, Chen J, Liu Y, Bao T. Identification of serum microRNAs in genome-wide serum microRNA expression profiles as novel noninvasive biomarkers for malignant peripheral nerve sheath tumor diagnosis. Med Oncol. 2013;30(2):531.

38. Luo J, Chen M, Huang H, et al. Circulating microRNA-122a as a diagnostic marker for hepatocellular carcinoma. Onco Targets Ther $2013 ; 6: 577-583$.

\section{Publish your work in this journal}

OncoTargets and Therapy is an international, peer-reviewed, open access journal focusing on the pathological basis of all cancers, potential targets for therapy and treatment protocols employed to improve the management of cancer patients. The journal also focuses on the impact of management programs and new therapeutic agents and protocols on

\section{Dovepress}

patient perspectives such as quality of life, adherence and satisfaction. The manuscript management system is completely online and includes a very quick and fair peer-review system, which is all easy to use. Visit http://www.dovepress.com/testimonials.php to read real quotes from published authors. 